CERN-TH-96/24

EFI-96-03

hep-ph/9602265

February 1996

\title{
ENHANCEMENT OF THE $\Lambda_{b}$ DECAY RATE \&
}

\author{
Jonathan L. Rosner \\ Div. TH, CERN \\ 1211 CH Geneva 23, Switzerland \\ and \\ Enrico Fermi Institute and Department of Physics \\ University of Chicago, Chicago, IL 60637
}

\begin{abstract}
The enhancement $\Delta \Gamma\left(\Lambda_{b}\right)$ of the $\Lambda_{b}$ decay rate due to four-fermion processes of weak scattering and Pauli interference is calculated within the quark model. An estimate of the relative bu wave function at zero separation, $|\Psi(0)|_{b u}^{2}$, is obtained in terms of the $\Sigma_{b}^{*}-\Sigma_{b}$ hyperfine splitting, the $B^{*}-B$ hyperfine splitting, and the $B$ meson decay constant $f_{B}$. For $M\left(\Sigma_{b}^{*}\right)-M\left(\Sigma_{b}\right)=56 \pm 16 \mathrm{MeV}, M\left(B^{*}\right)-M(B)=46 \mathrm{MeV}$, and $f_{B}=190 \pm 40$ $\mathrm{MeV}$, we find $\Delta \Gamma\left(\Lambda_{b}\right)=(0.025 \pm 0.013) \mathrm{ps}^{-1}$, to be compared with the observed enhancement $\Gamma\left(\Lambda_{b}\right)-\Gamma\left(B^{0}\right)=0.20 \pm 0.05 \mathrm{ps}^{-1}$. Even such a meager enhancement entails a value of $|\Psi(0)|_{b u}^{2}$ considerably larger than the corresponding value of $|\Psi(0)|_{c d}^{2}$ in the $\Lambda_{c}$ baryon.
\end{abstract}

PACS codes: 14.20.Mr, 14.20.Lq, 13.30.Eg, 12.39.Jh

\footnotetext{
${ }^{1}$ To be submitted to Phys. Lett. B.

${ }^{2}$ Permanent address.
} 
The differences among lifetimes of particles containing heavy quarks are expected to become smaller as the heavy quark mass increases and free-quark estimates become more reliable. Thus, although charmed particles have lifetimes ranging from less than $0.1 \mathrm{ps}$ for the $\Omega_{c}$ [1] to greater than 1 ps for the $D^{+}[2]$, mesons and baryons containing $b$ quarks are expected to have lifetimes differing no more than a few percent [3, 4, 5]. For example, it is expected that the process, $b u \rightarrow c d$ in the $\Lambda_{b}$ ("weak scattering"), when considered in conjunction with the partially offsetting process $b d \rightarrow c \bar{u} d d$ ("Pauli interference") should lead to a small enhancement in the $\Lambda_{b}$ decay rate, so that $\tau\left(\Lambda_{b}\right)=(0.9$ to 0.95$) \tau\left(B^{0}\right)$. Some caution has been urged with regard to the four-quark matrix element in these estimates [6]. In the present Letter we present a new evaluation of this matrix element which confirms the expected smallness of the enhancement. We perform this evaluation using a hyperfine splitting sensitive to the heavy quark - light quark interaction, which has recently become possible in $b$-flavored baryons as a result of a measurement of the $\Sigma_{b}^{*}-\Sigma_{b}$ splitting by the DELPHI Collaboration [7].

The observed $\Lambda_{b}$ lifetime is $\tau\left(\Lambda_{b}\right)=1.20 \pm 0.07 \mathrm{ps}$, while the $B^{0}$ decays more slowly: $\tau\left(B^{0}\right)=(1.58 \pm 0.05)$ ps. Here we have averaged a compilation of world data [8] (for which $\left.\tau\left(B^{0}\right)=1.18 \pm 0.07 \mathrm{ps}\right)$ with a new value [9] $\tau\left(B^{0}\right)=1.33 \pm 0.16 \pm 0.07 \mathrm{ps}$. The ratio of these two quantities is $\tau\left(\Lambda_{b}\right) / \tau\left(B^{0}\right)=0.76 \pm 0.05$, indicating an enhancement of the $\Lambda_{b}$ decay rate beyond the magnitude of usual estimates.

In the present Letter we find that, in spite of a large wave function for the bu pair in the initial baryon, which we denote by $|\Psi(0)|_{b u}^{2}$, only $(13 \pm 7) \%$ of the needed enhancement of the $\Lambda_{b}$ decay rate can be explained in terms of the effects of the four-fermion matrix element. (Isospin symmetry then dictates $|\Psi(0)|_{b d}^{2}=|\Psi(0)|_{b u}^{2}$.) If we assume wave functions are similar in all baryons with a single $b$ quark and two nonstrange quarks, this quantity can be related to the hyperfine splitting $M\left(\Sigma_{b}^{*}\right)-M\left(\Sigma_{b}\right)$, for which the DELPHI Collaboration at LEP [7] has recently quoted a large value of $56 \pm 16 \mathrm{MeV}$. We estimate the effect of gluon exchange by performing a similar calculation for $B$ mesons, relating the $B^{*}-B$ splitting to the $B$ meson decay constant and taking account of differing spin and hyperfine factors in the meson and baryon systems.

A relation for the enhancement of the $\Lambda_{c}$ decay rate due to the weak scattering process $c d \rightarrow s u$ was first pointed out in Ref. [10. At the same order in heavy quark mass, one must also take account of Pauli interference (interference between identical quarks in the final state) [11, 12]. Thus, for the $\Lambda_{b}$, one considers not only the process $b u \rightarrow c d$ (involving matrix elements between $\Lambda_{b}$ states of $(\bar{b} b)(\bar{u} u)$ operators), but also those processes involving matrix elements of $(\bar{b} b)(\bar{d} d)$ operators) which contribute to interference. The net result of four-quark operators in the $\Lambda_{b}$ is an enhancement of the decay rate by an amount (see, e.g., Refs. [11, 12, 5])

$$
\Delta \Gamma\left(\Lambda_{b}\right)=\frac{G_{F}^{2}}{2 \pi}|\Psi(0)|_{b u}^{2}\left|V_{u d}\right|^{2}\left|V_{c b}\right|^{2} m_{b}^{2}(1-x)^{2}\left[c_{-}^{2}-(1+x) c_{+}\left(c_{-}-c_{+} / 2\right)\right]
$$

Here we have neglected light-quark masses; $x \equiv m_{c}^{2} / m_{b}^{2}$, while $c_{-}$and $c_{+}=\left(c_{-}\right)^{-1 / 2}$ are the short-distance QCD enhancement and suppression factors for quarks in a color 
antitriplet and sextet, respectively: [13]:

$$
c_{-}=\left[\frac{\alpha_{s}\left(m_{c}^{2}\right)}{\alpha_{s}\left(M_{W}^{2}\right)}\right]^{\gamma} \quad, \quad \gamma=\frac{12}{33-2 n_{F}},
$$

with $n_{F}=5$ the number of active quark flavors between $m_{b}$ and $M_{W}$. The $c_{-}^{2}$ term in square brackets reflects the weak scattering process $b u \rightarrow c d \rightarrow b u$, while the remaining terms arise from destructive interference between the two intermediate $d$ quarks in the process $b d \rightarrow c \bar{u} d d \rightarrow b d$.

Taking the strong interaction scale in the modified-minimal-subtraction scheme for four quark flavors to be [14 $\Lambda \frac{(4)}{\mathrm{MS}}=200 \mathrm{MeV}$, we find $\alpha_{s}\left(m_{b}^{2}\right)=0.193$ and $\alpha_{s}\left(M_{W}^{2}\right)=$ 0.114 , and hence $c_{-}=1.32, c_{+}=0.87$. An estimate of $|\Psi(0)|_{b u}^{2}$ is then needed. We find it by comparing hyperfine splittings in mesons and baryons, under the assumption that the strength of the one-gluon exchange term is the same for the light quark - heavy quark pair in each system.

Our result, which we shall explain in more detail presently, is

$$
|\Psi(0)|_{b u}^{2}=2 \cdot \frac{2}{3} \cdot \frac{M\left(\Sigma_{b}^{*}\right)-M\left(\Sigma_{b}\right)}{M\left(B^{*}\right)-M(B)} \cdot \frac{M_{B} f_{B}^{2}}{12},
$$

where the first factor relates to color, the second to spin, and the last term is the nonrelativistic estimate of the $b \bar{u}$ wave function in the $B$ meson [15]. (Here one may use the spin-averaged value of vector and pseudoscalar masses for $M_{M}$.) With the DELPHI value of $M\left(\Sigma_{b}^{*}\right)-M\left(\Sigma_{b}\right)$, the $B^{*}-B$ splitting of $46 \mathrm{MeV}$ [2], and the estimate [16, 17] $f_{B}=190 \pm 40 \mathrm{MeV}$, we obtain $|\Psi(0)|_{b u}^{2}=(2.6 \pm 1.3) \times 10^{-2} \mathrm{GeV}^{3}$. This is to be compared with $|\Psi(0)|_{b \bar{u}}^{2}=M_{B} f_{B}^{2} / 12=(1.6 \pm 0.7) \times 10^{-2} \mathrm{GeV}^{3}$ for the $B$ meson. Our assumption of equal values of $\alpha_{s}$ governing the hyperfine interaction of the light - heavy pair in the meson and baryon must be viewed cautiously in this light. It is possible that $\alpha_{s}$ in the more compact baryonic system (since the wave function appears to be larger) is smaller than in the meson, which would further enhance the estimate of $|\Psi(0)|_{b u}^{2}$.

Several previous estimates of the four-quark matrix element (see, e.g., Refs. [1, 10, 11, 18]) utilized the hyperfine splitting between the $\Lambda_{b}$ and the $\Sigma_{b}$ (or the corresponding charmed states). Since this splitting involves the interaction of two light quarks as well as one light and one heavy quark, it necessarily involves a statement about light quark masses, as well as about the relation between the relative wave function of two light quarks and that of a light and a heavy quark. Our approach avoids such assumptions.

In the relation (11) we now neglect $\sin \theta_{c}$ (setting $\left.V_{u d}=1\right)$, and choose $m_{b}=5.1 \mathrm{GeV}$, $m_{c}=1.7 \mathrm{GeV}$, and $\left|V_{c b}\right|=0.040 \pm 0.003$. We then find

$$
\Delta \Gamma\left(\Lambda_{b}\right)=0.025 \pm 0.013 \mathrm{ps}^{-1}
$$

The decay rates of the $B^{0}$ and $\Lambda_{b}$ are $\Gamma\left(B^{0}\right)=0.63 \pm 0.02 \mathrm{ps}^{-1}$ and $\Gamma\left(\Lambda_{b}\right)=0.83 \pm 0.05$ $\mathrm{ps}^{-1}$, differing by $\Delta \Gamma\left(\Lambda_{b}\right)=0.20 \pm 0.05 \mathrm{ps}^{-1}$. The four-quark processes noted above can explain only $(13 \pm 7) \%$ of this difference, leading to an enhancement of only $(4 \pm 2) \%$ of the total $\Lambda_{b}$ decay rate in contrast with the needed enhancement of $(32 \pm 8) \%$. 
We now give some details of the calculation and see how well it does for the $\Lambda_{c}$.

The hyperfine interaction in a meson $M_{i \bar{j}}$ composed of a quark $i$ and an antiquark $\bar{j}$ leads to a mass shift [19]

$$
\Delta M\left(M_{i j}\right)=\frac{32 \pi}{9} \alpha_{s} \frac{\left\langle\hat{s}_{i} \cdot \hat{s}_{j}\right\rangle}{m_{i} m_{j}}|\Psi(0)|^{2}
$$

where $\hat{s}_{i}$ refers to a quark spin operator. The corresponding result for a baryon $B_{i j k}$ is

$$
\Delta M\left(B_{i j k}\right)=\frac{16 \pi}{9} \alpha_{s} \sum_{i>j} \frac{\left\langle\hat{s}_{i} \cdot \hat{s}_{j}\right\rangle}{m_{i} m_{j}}|\Psi(0)|_{i j}^{2} \quad .
$$

The relative factor of 2 arises from the different color factors for a quark and antiquark in a meson (a triplet and antitriplet making a singlet) and two quarks in a baryon (two triplets making an antitriplet). This factor of 2 is the first term in Eq. (3).

We take the ratio of hyperfine splittings in mesons and baryons so that light quark masses and values of $\alpha_{s}$ cancel out. We thus assume that (a) effective light quark masses in mesons and baryons are equal (borne out at the $20 \%$ level by phenomenological fits to meson and baryon spectra [19]), and (b) the values of $\alpha_{s}$ governing the corresponding hyperfine splittings are similar (reasonable since in both mesons and baryons one is concerned with systems of one light and one heavy quark).

We must isolate a baryon mass shift sensitive to the interaction between a light quark and a heavy one. The $\Sigma_{b}^{*}-\Sigma_{b}$ splitting is the appropriate quantity. In both $\Sigma_{b}$ and $\Sigma_{b}^{*}$, the light quarks are coupled up to spin 1. The splitting then depends purely on the light quark - heavy quark interaction.

The wave function between a light quark and a heavy one is assumed, as mentioned, to be identical in the $\Lambda_{b}$ and in the $\Sigma_{b}-\Sigma_{b}^{*}$ system. The two light quarks are coupled up to zero spin in the $\Lambda_{b}$, and hence have zero net hyperfine intreraction with the heavy quark, while the hyperfine interaction between the light quarks and the heavy one averages to zero if we take the spin-weighted average of the $\Sigma_{b}$ and the $\Sigma_{b}^{*}$.

The value of $\left\langle\hat{s}_{Q} \cdot \hat{s}_{\bar{q}}\right\rangle$ is $(1 / 4,-3 / 4)$ for a $\left({ }^{3} S_{1},{ }^{1} S_{0}\right) Q \bar{q}$ meson, where $Q$ and $q$ are the heavy and light quark. For a baryon $Q q q$ with $S_{q q}=1$, one has $\left\langle\hat{s}_{Q} \cdot \hat{s}_{q}\right\rangle=(1 / 4,-1 / 2)$ for states with total spin $(3 / 2,1 / 2)$. Thus the difference in $\hat{s}_{i} \cdot \hat{s}_{j}$ for the $\Sigma_{b}^{*}-\Sigma_{b}$ splitting (counting a factor of 2 for the two light quarks in the baryons) is $3 / 2$ that for the $B^{*}-B$ splitting. The factor of $2 / 3$ in Eq. (3) compensates for this ratio.

The relation [15] $|\Psi(0)|^{2}=M_{M} f_{M}^{2} / 12$ for the square of the wave function of a $Q \bar{q}$ meson probably has important corrections of order $1 / m_{Q}$, if lattice calculations are any guide [17]. These are ignored in the present discussion. They are likely to be more important when we apply the present method to charmed states.

The corresponding calculation for charmed particles makes use of the following inputs.

1. The $D$ meson decay constant was taken [16] to be $f_{D}=240 \pm 40 \mathrm{MeV}$, leading (with $\left.M_{D}=1973 \mathrm{MeV}\right)$ to $|\Psi(0)|_{c \bar{d}}^{2}=(0.95 \pm 0.32) \times 10^{-2} \mathrm{GeV}^{3}$.

2. The $D^{*}-D$ splitting is assumed to be $141 \mathrm{MeV}$ (the average for charged and neutral states [2]). 
Table I: Comparison of predicted squares of wave functions and decay rate enhancements for $\Lambda_{c}$ and $\Lambda_{b}$.

\begin{tabular}{ccc}
\hline Quantity (units) & Charm & Beauty \\
\hline$f_{M}(\mathrm{MeV})$ & $240 \pm 40$ & $190 \pm 40$ \\
$|\Psi(0)|_{Q \bar{q}}^{2}\left(10^{-2} \mathrm{GeV}^{3}\right)$ & $0.95 \pm 0.32$ & $1.6 \pm 0.7$ \\
$M\left({ }^{3} S_{1}\right)-M\left({ }^{1} S_{0}\right)(\mathrm{MeV})$ & 141 & 46 \\
$M\left(\Sigma^{*}\right)-M(\Sigma)(\mathrm{MeV})$ & $77 \pm 7$ & $56 \pm 16$ \\
$|\Psi(0)|_{Q q}^{2}\left(10^{-2} \mathrm{GeV}^{3}\right)$ & $0.69 \pm 0.24$ & $2.6 \pm 1.3$ \\
$c_{-}$ & 1.60 & 1.32 \\
$c_{+}$ & 0.79 & 0.87 \\
$c_{-}^{2}-(1+x) c_{+}\left(c_{-}-c_{+}^{2} / 2\right)$ & 1.52 & 0.88 \\
$\Delta \Gamma\left(\Lambda_{Q}\right)\left(\mathrm{ps}^{-1}\right)$ & $0.8 \pm 0.3$ & $0.025 \pm 0.013$ \\
\hline
\end{tabular}

3. Charmed baryon masses are taken to be $M\left(\Sigma_{c}\right)=2453 \mathrm{MeV}$ [2] and $M\left(\Sigma_{c}^{*}\right)=$ $2530 \pm 7 \mathrm{MeV}$ [20].

4. The strong fine-structure-constant at $m_{c}^{2}$ is taken to be $\alpha_{s}\left(m_{c}^{2}\right)=0.289$, consistent with the QCD scale mentioned above, leading to $c_{-}=1.60, c_{+}=0.79$.

5. The strange quark mass is taken to have a typical constituent-quark value, $m_{s}=$ $0.5 \mathrm{GeV}$. We continue to neglect $u$ and $d$-quark masses for simplicity.

6. The CKM factors in Eq. (11) undergo the replacements $\left|V_{u d}\right|^{2}\left|V_{c b}\right|^{2} \rightarrow\left|V_{c s}\right|^{2}\left|V_{u d}\right|^{2}$, which we approximate by 1 (again neglecting $\sin \theta_{c}$ ).

The resulting matrix element $|\Psi(0)|_{c d}^{2}=(0.69 \pm 0.24) \times 10^{-2} \mathrm{GeV}^{3}$ is consistent with an estimate in Ref. [10] of $|\Psi(0)|_{c d}^{2}=0.74 \times 10^{-2} \mathrm{GeV}^{3}$. In that work, the value of $|\Psi(0)|_{c d}^{2}$ was assumed to be the same as for two light quarks in the charmed baryon, and was estimated using the observed $\Sigma_{c}-\Lambda_{c}$ splitting. It was also necessary to assume a specific value of $\alpha_{s}=0.58$ in the hyperfine interaction expression (6), which we do not do here. Our value of $c_{-}$is smaller than assumed in Ref. "10 and we take account of destructive interference, leading to a smaller result for $\Delta \Gamma\left(\Lambda_{c}\right)$.

The results for systems with $c$ and $b$ quarks based on our method are summarized in Table I. Several remarks can be made.

(a) The difference between the central values of $|\Psi(0)|_{Q \bar{q}}^{2}$ for charm and beauty reflects the likely importance of $1 / m_{Q}$ corrections (see, e.g., Ref. [17]), or - in the language of the quark model - of reduced mass effects.

(b) The $\Sigma_{c}^{*}-\Sigma_{c}$ hyperfine splitting used in this calculation is based on one claim for observation of the $\Sigma_{c}^{*}$ [20], which requires confirmation.

(c) The value of $|\Psi(0)|_{b u}^{2}$ is somewhat large in comparison with the others for lightheavy systems. It would be helpful to verify the large hyperfine splitting between $\Sigma_{b}^{*}$ and $\Sigma_{b}$ claimed by the DELPHI Collaboration [7]. The ratio of hyperfine splittings 
for charmed and beauty mesons is approximately $3: 1$, as expected if these splittings scale as $1 / m_{Q}$. In contrast, the corresponding ratio for baryons is considerably smaller, indicating a violation of $1 / m_{Q}$ scaling.

(d) The enhancement of the $\Lambda_{c}$ decay rate is quite modest. With $\Gamma\left(\Lambda_{c}\right) \approx 5 \mathrm{ps}^{-1}$, to be compared with $\Gamma\left(D^{0}\right) \approx 2.4 \mathrm{ps}^{-1}$ and $\Gamma\left(D^{+}\right) \approx 1 \mathrm{ps}^{-1}$, one seeks an enhancement of at least $\Gamma\left(\Lambda_{c}\right)-\Gamma\left(D^{0}\right) \approx 2.6 \mathrm{ps}^{-1}$. If the enhancements $\Delta \Gamma\left(\Lambda_{Q}\right)$ in Table I were about a factor of 4 larger, we could accommodate both the $\Lambda_{c}$ and $\Lambda_{b}$ decay rates, but this is not consistent with our estimates of the matrix elements and their effects on decay rates. In particular, the effect of Pauli interference is to cut the naïve estimate of the enhancement due to weak scattering alone [10] by roughly a factor of 2 . Hybrid logarithms [11], not considered here, have a relatively modest effect, leading if anything to further suppression of the enhancement for $\Lambda_{b}$ decay [5].

To summarize, we have used the hyperfine splitting between $\Sigma_{b}^{*}$ and $\Sigma_{b}$ claimed by the DELPHI Collaboration [7] to estimate the overlap of quark wave functions between the $b$ quark and the light quarks in the $\Lambda_{b}$, and hence to estimate the effect of four-quark operators on its decay rate. Even though the matrix element $|\Psi(0)|_{b u}^{2}=|\Psi(0)|_{b d}^{2}$ deduced from the DELPHI result is quite large on the scale of those for heavy-light systems, one can only account for $(13 \pm 7) \%$ of the difference between the $\Lambda_{b}$ and $B^{0}$ decay rates, or an enhancement of $(4 \pm 2) \%$ of the $\Lambda_{b}$ decay rate. A similar approach also falls short of accounting for the corresponding enhancement for the $\Lambda_{c}$ decay rate. If the enhanced $\Lambda_{b}$ decay rate is borne out by further data, we can only speculate that strong final-state interactions which cannot be anticipated on the basis of perturbative QCD must play a role even at the rather high mass of the $\Lambda_{b}$.

I wish to thank G. Martinelli, M. Neubert, C. T. Sachrajda, M. Shifman, and N. G. Uraltsev for fruitful discussions, and the Physics Department of the Technion for its hospitality during the initial stages of this investigation. This work was supported in part by the United States - Israel Binational Science Foundation under Research Grant Agreement 94-00253/1 and by the United States Department of Energy under Contract No. DE FG02 90ER40560.

\section{References}

[1] Fermilab E687 Collaboration, P. L. Frabetti et al., Phys. Lett. B 357 (1995) 678; CERN WA89 Collaboration, M. I. Adamovich et al., Phys. Lett. B 358 (1995) 151.

[2] Particle Data Group, L. Montanet et al., Phys. Rev. D 50 (1994) 1173.

[3] I. I. Bigi et al., in B Decays, edited by S. Stone (World Scientific, Singapore, 1994), p. 132.

[4] I. I. Bigi, University of Notre Dame report UND-HEP-95-BIG02, hep-ph/9508408, preliminary version of a paper to be submitted to Physics Reports.

[5] M. Neubert and C. T. Sachrajda, CERN report CERN-TH/96-19, February, 1996, in preparation. 
[6] M. Shifman, presented at Symposium on Heavy Flavours, Haifa, Israel, December, 1995 (unpublished).

[7] DELPHI Collaboration, P. Abreu et al., DELPHI report DELPHI 95-107 PHYS 542, June, 1995, presented by M. Feindt, C. Kreuter, A. Miagkov, and O. Podobrin at EPS-HEP 95 Conference, Brussels, 27 July - 2 August, 1995 (unpublished).

[8] V. Sharma, presented at Symposium on Heavy Flavours, Haifa, Israel, December, 1995 (unpublished), updated version of V. Sharma and F. V. Weber, in B Decays, edited by S. Stone (World Scientific, Singapore, 1994), p. 395.

[9] CDF Collaboration, F. Abe et al., 1996 (unpublished).

[10] V. Barger, J. P. Leveille, and P. M. Stevenson, Phys. Rev. Lett. 44 (1980) 226.

[11] M. B. Voloshin and M. A. Shifman, Yad. Fiz. 41 (1985) 187 [Sov. J. Nucl. Phys. 41 (1985) 120]; Zh. Eksp. Teor. Fiz. 91 (1986) 1180 [Sov. Phys. - JETP 64 (1986) 698]; M. B. Voloshin, N. G. Uraltsev, V. A. Khoze, and M. A. Shifman, Yad. Fiz. 46 (1987) 181 [Sov. J. Nucl. Phys. 46 (1987) 112].

[12] N. Bilić, B. Guberina, and J. Trampetić, Nucl. Phys. B248 (1984) 261; B. Guberina, R. Rückl, and J. Trampetić, Zeit. Phys. C 33 (1986) 297.

[13] G. Altarelli and L. Maiani, Phys. Lett. 52B (1974) 351; M. K. Gaillard and B. W. Lee, Phys. Rev. Lett. 33 (1974) 108; G. Altarelli, N. Cabibbo, and L. Maiani, Phys. Lett. 57B (1975) 277.

[14] W. Kwong, P. B. Mackenzie, R. Rosenfeld, and J. L. Rosner, Phys. Rev. D 37 (1988) 3210.

[15] E. V. Shuryak, Nucl. Phys. B198 (1982) 83.

[16] J. L. Rosner, Enrico Fermi Institute Report No. EFI 95-36, lectures presented at the VIII J. A. Swieca Summer School, Rio de Janeiro, Brazil, February 7 - 11, 1995, Proceedings to be published by World Scientific.

[17] UKQCD Collaboration, R. M. Baxter et al., Phys. Rev. D 49 (1994) 1594; C. W. Bernard, J. N. Labrenz, and A. Soni, ibid. 49 (1994) 2536; A. Duncan et al., ibid. 51 (1995) 5101.

[18] B. Blok and M. Shifman, in Proceedings of the Third Workshop on Physics at a TauCharm Factory, Marbella, Spain, June 1993, edited by J. Kirkby and R. Kirkby (Editions Frontières, Gif-sur-Yvette, France, 1994).

[19] A. De Rújula, H. Georgi, and S. L. Glashow, Phys. Rev. D 12 (1975) 147.

[20] V. A. Ammosov et al., Pis'ma Zh. Eksp. Teor. Fiz. 58 (1993) 241 [JETP Lett. 58 (1993) 247]. 